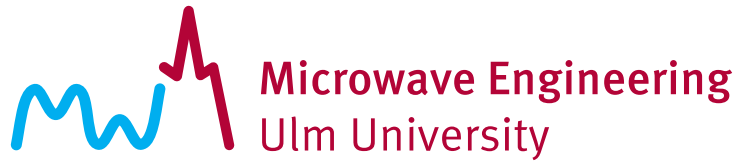

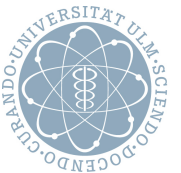

\section{On the Calibration of mm-Wave MIMO Radars Using Sparse Antenna Arrays for DoA Estimation}

André Dürr, Dominik Schwarz, Fabian Roos, Philipp Hügler, Stephan Bucher, Patrik Grüuner, and Christian Waldschmidt

(C) 2019 IEEE. Personal use of this material is permitted. Permission from IEEE must be obtained for all other uses, in any current or future media, including reprinting/republishing this material for advertising or promotional purposes, creating new collective works, for resale or redistribution to servers or lists, or reuse of any copyrighted component of this work in other works.

DOI: $10.23919 /$ EuMC.2019.8910838 


\title{
On the Calibration of mm-Wave MIMO Radars Using Sparse Antenna Arrays for DoA Estimation
}

\author{
André Dürr ${ }^{1}$, Dominik Schwarz, Fabian Roos, Philipp Hügler, \\ Stephan Bucher, Patrik Grüner, and Christian Waldschmidt \\ Institute of Microwave Engineering, Ulm University, 89081 Ulm, Germany \\ 1 andre.duerr@uni-ulm.de
}

\begin{abstract}
The multiple-input and multiple-output (MIMO) principle is a well-established method to improve the angular resolution of radars. Due to hardware limitations the maximum number of transmit and receive channels is often limited. In order to further increase the angular resolution with a given number of hardware channels, sparse arrays can be employed. However, the optimal placement of the antenna positions is a critical design parameter and it is difficult to consider non-idealities and mutual coupling within the design process. This paper addresses the non-idealities and the influence of coupling on the virtual array positions of a MIMO radar. An efficient calibration method to determine the real antenna positions is proposed. The discrete Fourier transform (DFT) based direction-of-arrival (DoA) estimation is shown with measurements of a $77 \mathrm{GHz}$ and a $160 \mathrm{GHz}$ MIMO radar for a uniform linear array (ULA) and two sparse arrays in comparison.

Keywords - MIMO radar, antenna coupling, calibration, direction-of-arrival estimation, sparse array, automotive radar
\end{abstract}

\section{INTRODUCTION}

In the last decade, MIMO radars gained a strong interest to enhance the angular resolution of radars thanks to their increased virtual aperture exceeding the physical one $[1,2]$. However, the number of transmit and receive antennas to form a virtual aperture is often restricted. In order to further enhance the angular resolution with a given number of hardware channels, sparse arrays can be realized.

Strategies for the optimum placement of the antennas in sparse arrays have extensively been discussed [3-5]. These design strategies do not consider non-idealities and mutual coupling within the design process, resulting in a degradation of DoA estimation performance [6].

This paper compares the antenna position shifts due to coupling and non-idealities for ULAs and sparse arrays with different hardware realizations and different center frequencies. The influence on the DoA estimation for a ULA and two sparse arrays at $77 \mathrm{GHz}$ and $160 \mathrm{GHz}$ is investigated. Furthermore, an efficient calibration method to extract the true antenna positions is proposed, which coincide with the electrical behavior of the array. The result can be used in an efficient DFT-based angle estimation algorithm, which is experimentally verified for all three arrays.

\section{Antenna Positions And ERror Sources}

In order to distinguish the different error sources, the following definitions for the antenna positions are used:

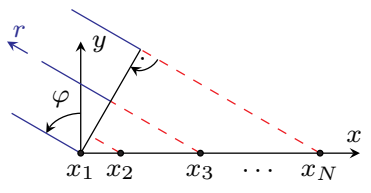

Fig. 1. Reference system for a target at distance $r$ and angle $\varphi$ in the far-field of the array with element positions $x_{k}$. The steering path differences are highlighted in dashed red.

Ideal antenna positions are the outcome of the array design. To maintain the system performance, only small deviations from these ideal positions are typically tolerated.

Mechanical antenna positions are the physical element positions on the fabricated antenna array. The deviation between the ideal and mechanical antenna positions depends on the array fabrication process. Printed circuit board (PCB) manufacturing tolerances only cause small mechanical antenna position shifts. For more complicated antenna systems such as distributed PCBs, separate antennas, or radar transceivers with on-chip antennas, mechanical placement tolerances have to be taken into consideration.

Electrical antenna positions describe the positions corresponding to the electrical behavior of the array. Differences between the electrical and mechanical positions occur due to coupling, non-ideal radiation patterns as well as displacements in the phase centers of the antenna elements.

\section{SIMULATION: INFLUENCE OF COUPLING}

For a reference system as depicted in Fig. 1 the steering vector $\mathbf{v}(\varphi)$ of the virtual receive array can be written as

$$
\mathbf{v}(\varphi)=\left[\begin{array}{c}
1 \\
\mathrm{e}^{\mathrm{j}\left(\frac{2 \pi}{\lambda_{0}}\left(x_{2}-x_{1}\right) \sin \varphi+\psi_{2}\right)} \\
\vdots \\
\mathrm{e}^{\mathrm{j}\left(\frac{2 \pi}{\lambda_{0}}\left(x_{N_{\mathrm{VX}}}-x_{1}\right) \sin \varphi+\psi_{N_{\mathrm{VX}}}\right)}
\end{array}\right],
$$

with the element positions $x_{k}$, the phase offset $\psi_{k}$ due to different transmission line lengths and variances of the RF components, the wavelength $\lambda_{0}$, and the virtual channel number $N_{\mathrm{VX}}$. The virtual element position vector $\mathbf{x}$ is

$$
\mathbf{x}=\left[x_{\mathrm{TX}_{1} \mathrm{RX}_{1}}, \ldots, x_{\mathrm{TX}_{1} \mathrm{RX}_{\mathrm{N}}}, x_{\mathrm{TX}_{2} \mathrm{RX}_{1}}, \ldots, x_{\mathrm{TX}_{\mathrm{N}} \mathrm{RX}_{\mathrm{N}}}\right]^{T},
$$

where $(\cdot)^{T}$ describes the transpose. By considering a set of steering vectors for $N$ different DoAs, the virtual steering matrix $\mathbf{V}=\left[\mathbf{v}\left(\varphi_{1}\right) \cdots \mathbf{v}\left(\varphi_{N}\right)\right]$ is formed. 


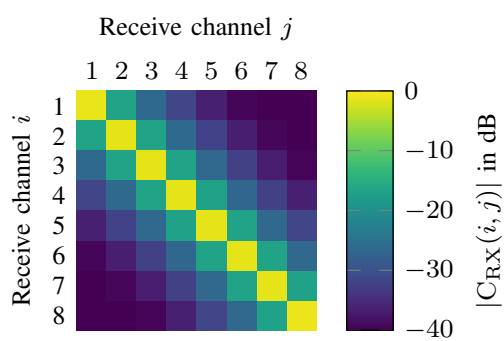

(a) RX coupling magnitude

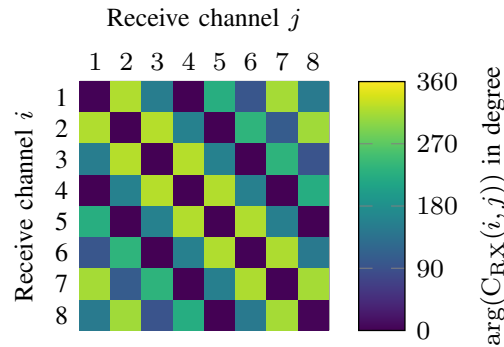

(b) RX coupling phase

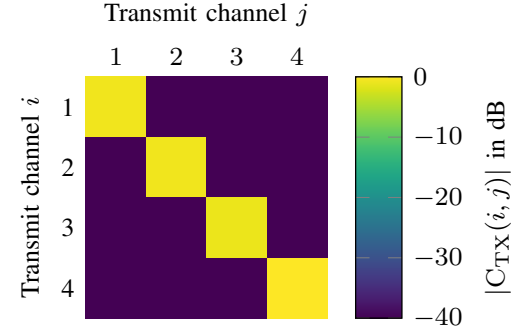

(c) TX coupling magnitude

Fig. 2. Simulated coupling matrices for the receive $(a, b)$ and the transmit antennas (c) for the $4 \times 8$ ULA.

The effect of coupling on the incoming signals $\mathbf{s}$ can be described by $[7,8]$

$$
\begin{gathered}
\mathbf{y}=\mathbf{V}_{\mathrm{C}} \mathbf{s}+\mathbf{w}, \\
\text { with } \quad \mathbf{V}_{\mathrm{C}}=\mathbf{C V},
\end{gathered}
$$

where $\mathbf{y}$ denotes the receiver output signal vector, $\mathbf{V}_{\mathrm{C}}$ the coupled steering matrix and $\mathbf{w}$ white Gaussian noise. The ideal signal model of (1) is, therefore, extended by the coupling matrix C. Without coupling effects, the matrix is the identity matrix. With coupling, the entry $\mathrm{C}(i, j)$ describes the coupling from antenna $j$ to antenna $i$.

If the virtual channels are sorted as defined in (2), the virtual coupling matrix for the transmit-receive combination is given by

$$
\mathbf{C}=\mathbf{C}_{\mathrm{TX}} \otimes \mathbf{C}_{\mathrm{RX}}
$$

where $\otimes$ denotes the Kronecker tensor product. This can be interpreted as the additive influence of the receiver coupling to all coupled transmit signals.

In order to determine the Z-parameters, full-wave field simulations of the transmit and receive array are performed for the fabricated $4 \times 8$ ULA at $77 \mathrm{GHz}$ with $\lambda_{0} / 2$ spacing and non-overlapping elements. Afterwards, the coupling matrices $\mathbf{C}_{\mathrm{RX}}$ and $\mathbf{C}_{\mathrm{TX}}$ are calculated from the Z-parameters, as described in [9]. The results are shown in Fig. 2.

At the receiver, the coupling of adjacent channels is identical in both the magnitude and phase, see Fig. 2(a) and (b). For the transmitters with a spacing of $4 \lambda_{0}$, the coupling is low and can be neglected, see Fig. 2(c).

The position deviations between the ideal and the electrical positions - derived from the coupled and ideal non-coupled steering matrix - are shown in Fig. 3 for the considered $4 \times 8$ ULA. The first 8 virtual elements belong to the first transmitter. Only the edge elements, i.e., the virtual channels 1 and 8 , are affected by an asymmetric coupling to the adjacent receivers, see Fig. 2. In comparison, the coupling of the center elements is symmetric and only small deviations from the ideal element positions occur. This pattern is repeated for all transceivers. If more antennas are used within the ULA, the percentage of highly affected elements by an electrical antenna position shift decreases.

Full-wave simulations are time-consuming and it is almost not possible to consider all non-idealities. Thus, a simple

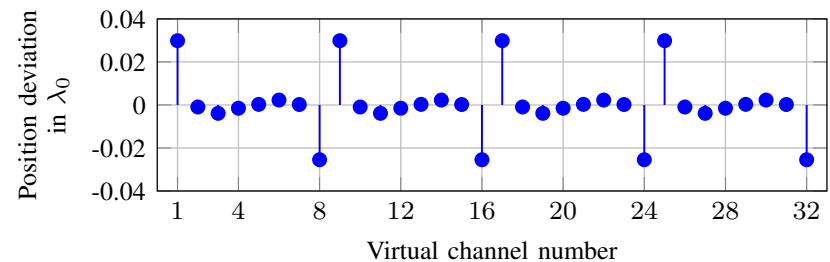

Fig. 3. Influence of the simulated coupling matrix on the element positions of the virtual channels.

calibration method which determines the electrical behavior of the antennas is desirable.

\section{Calibration Method}

To determine the constant phase term $\psi_{k}$ in (1), radar systems always need to be calibrated.

\section{A. Calibration Setup}

In order to calibrate a radar system, a point-like target is positioned in the far-field of the antenna array under $\varphi=0^{\circ}$. This calibration can be conducted angle-dependently by rotating the antenna array around its antenna array center point. Alternatively, the radar stays fixed and the calibration object is rotated along a spherical trajectory around the radar. Using such a calibration method, it is possible to determine the phase term according to the signal model of (1).

Fig. 4 shows the phase progressions of the $4 \times 8$ ULA derived from a full calibration for the azimuth angles from $-90^{\circ}$ to $90^{\circ}$ with an angular step size of $0.5^{\circ}$. They are exemplarily shown for the virtual receive channels belonging to the first transmitter and are related to the first virtual channel.

This calibration method requires 361 measurements. The angular step size depends on the array size $d_{A}=x_{N_{\mathrm{VX}}}-x_{1}$ and is chosen such that the absolute phase difference between two calibration points is much smaller than $\pi$. Due to its large number of required single snapshot measurements, this calibration is time-consuming.

\section{B. Electrical Antenna Positions: A Faster Calibration}

Fig. 4 shows that the relative phase progressions for the virtual receive channels belonging to the first transmitter are linearly dependent on $\sin (\varphi)$ assuming that the coupling does 


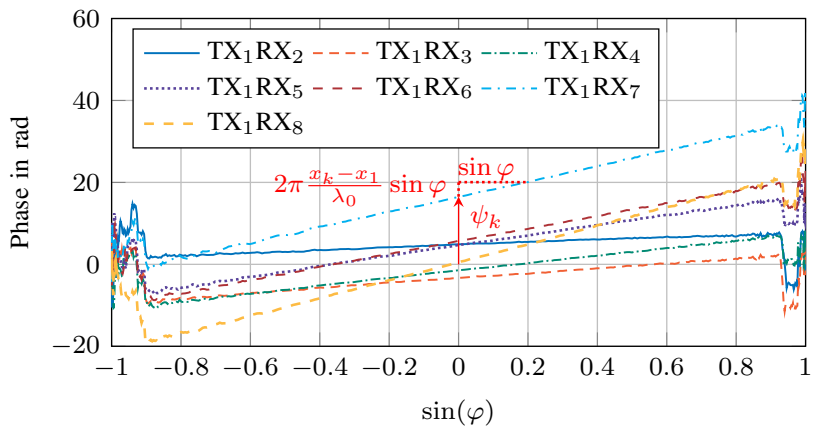

Fig. 4. Measurement of the phase progression of the $4 \times 8$ ULA relative to the first virtual channel as a function of the calibration angle for the virtual receive channels belonging to the first transmitter.

not dependent on $\varphi$. This can be expressed according to the signal model in (1) for the $k$-th virtual channel by

$$
\Delta \psi_{k, 1}=\frac{2 \pi}{\lambda_{0}} x_{k, 1} \sin (\varphi)+\psi_{k},
$$

with the virtual antenna distance $x_{k, 1}=x_{k}-x_{1}$ relative to the virtual receive antenna 1 .

Thus, it is basically sufficient to conduct the calibration for a limited angular range, which reduces the number of required calibration steps significantly. In the following, the approximation was done exemplarily for the angular range $\varphi \in\left[-11.5^{\circ} ; 11.5^{\circ}\right] \Rightarrow \sin (\varphi) \in[-0.2 ; 0.2]$. For the same angular step size of $0.5^{\circ}$, the calibration effort is reduced from 361 to 47 calibration measurements.

The linear equation (6) can be expressed for $L$ calibration measurements and for the $k$-th virtual channel in matrix notation by

$$
\left[\begin{array}{c}
\Delta \psi_{k, 1} \\
\vdots \\
\Delta \psi_{k, L}
\end{array}\right]=\left[\begin{array}{cc}
\sin \left(\varphi_{1}\right) & 1 \\
\vdots & \vdots \\
\sin \left(\varphi_{L}\right) & 1
\end{array}\right]\left[\begin{array}{c}
\frac{2 \pi}{\lambda_{0}} x_{k, 1} \\
\psi_{k}
\end{array}\right] .
$$

Applying a matrix inversion the constant phase term $\psi_{k}$ and the relative virtual antenna position $x_{k, 1}$ in (7) can be approximated with a least-squares solution. This coincides with the electric virtual antenna positions including all manufacturing tolerances and mutual coupling effects.

\section{Electrical Antenna Position Deviation for Sparse Arrays}

The calibration method is now applied to a ULA at $77 \mathrm{GHz}$ and to sparse arrays at $77 \mathrm{GHz}$ and $160 \mathrm{GHz}$. While the $77 \mathrm{GHz}$ arrays are realized with PCB antennas, the $160 \mathrm{GHz}$ sparse array uses on-chip antennas on multiple chips. Thus, deviations of mechanical antenna positions can only be neglected for the ULA and the sparse array at $77 \mathrm{GHz}$. The positions of the ideal virtual elements of the considered arrays are depicted in Fig. 5. In comparison to the ULA, the virtual antenna elements for the sparse arrays are non-uniformly distributed and interleaved if referred to the individual MIMO transmit antennas. Additionally, the array size is increased significantly.

The simulations presented in Section III show that for the ULA the coupling only has a minor impact on the electrical

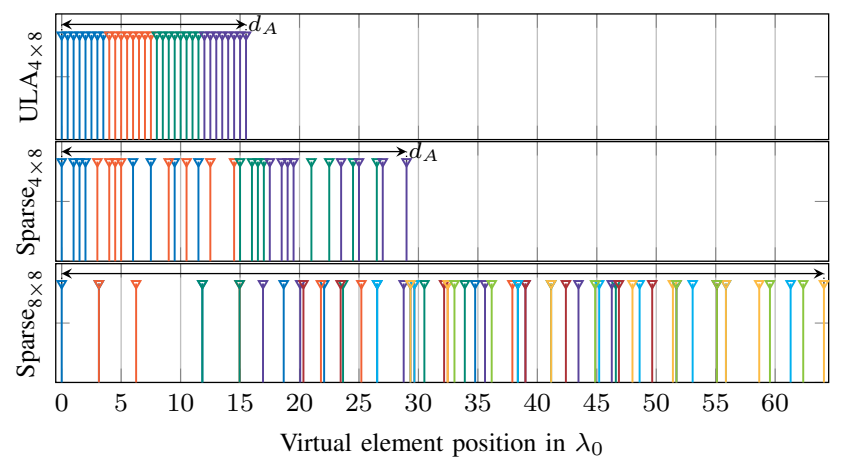

Fig. 5. Ideal virtual element positions of the $4 \times 8$ ULA and the $4 \times 8$ sparse array at $77 \mathrm{GHz}$ and the $8 \times 8$ sparse array at $160 \mathrm{GHz}$.

$\nabla \mathrm{TX}_{1} \mathrm{RX}_{i} ; \quad \nabla \mathrm{TX}_{2} \mathrm{RX}_{i} ; \quad \nabla \mathrm{TX}_{3} \mathrm{RX}_{i} ; \quad \nabla \quad \mathrm{TX}_{4} \mathrm{RX}_{i}$,

$\nabla \mathrm{TX}_{5} \mathrm{RX}_{i} ; \quad \nabla \quad \mathrm{TX}_{6} \mathrm{RX}_{i} ; \quad \nabla \quad \mathrm{TX}_{7} \mathrm{RX}_{i} ; \quad \nabla \quad \mathrm{TX}_{8} \mathrm{RX}_{i}$.

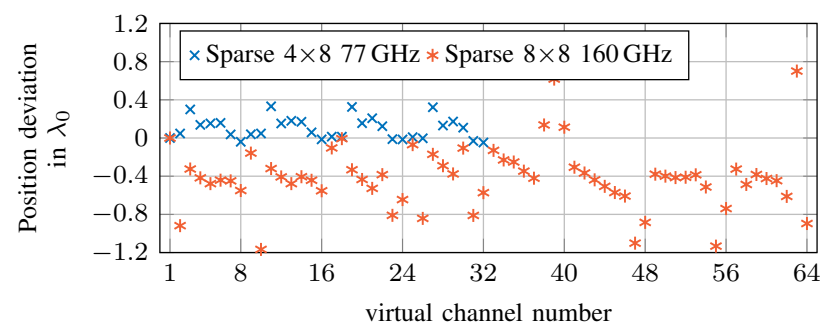

Fig. 6. Measured deviation of the virtual element positions for the $77 \mathrm{GHz}$ and the $160 \mathrm{GHz}$ sparse array. The electrical antenna positions are calculated with the proposed calibration algorithm.

virtual antenna positions due to its uniform coupling matrix. For the sparse arrays the electrical antenna positions are determined using the calibration method of Section IV and compared with the theoretical ideal virtual antenna positions. The deviations between the ideal and the electrical virtual antenna positions are shown in Fig. 6. The calibration of the $77 \mathrm{GHz}$ array proves that non-idealities and coupling effects shift the electrical virtual antenna element positions distinctly. By comparison, the $160 \mathrm{GHz}$ array is also affected by mechanical tolerances resulting in larger deviations.

\section{DFT FOR DOA ESTIMATION}

If the electrical antenna positions $x_{k}$ and the phase offsets $\psi_{k}$ are known, it is possible to obtain the angular spectrum, i.e., the vector $\mathbf{P}(\varphi)$ by a DFT, where the $n$-th entry $P_{n}$ is given as

$$
\begin{aligned}
P_{n} & =\sum_{k=1}^{N_{\mathrm{VX}}} y_{k} \mathrm{e}^{-\mathrm{j} \psi_{k}} \mathrm{e}^{-\mathrm{j} 2 \pi \frac{k^{\prime}}{N_{\mathrm{ZP}}} n}, \\
\text { with } \quad k^{\prime} & =\frac{x_{k}-x_{1}}{\lambda_{0} / 2}, \\
N_{\mathrm{ZP}} & =\frac{180^{\circ}}{\Delta \varphi} .
\end{aligned}
$$

The measurement vector $\mathbf{y}=\left[y_{1}, \ldots, y_{N_{\mathrm{Vx}}}\right]$ applied in (8) contains the spatial samples of the virtual antenna elements on the incoming signal across the array with the index $k \in\left[1, N_{\mathrm{VX}}\right]$ after range-Doppler processing. It is corrected 


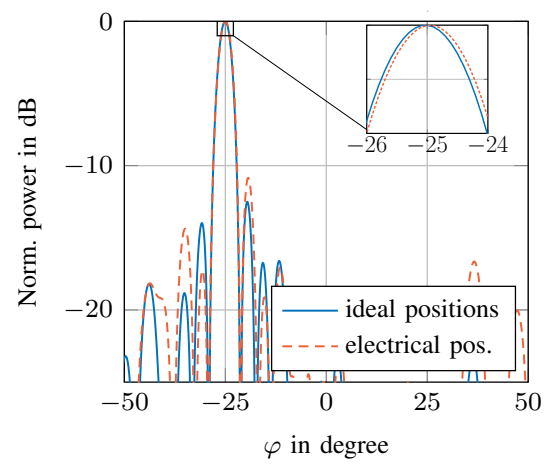

(a) $4 \times 8$ ULA $(77 \mathrm{GHz})$

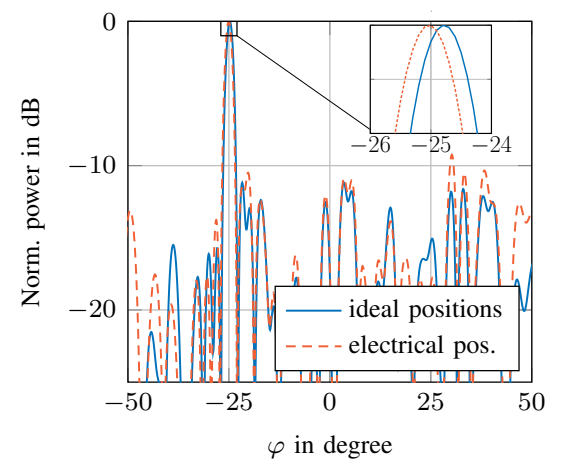

(b) $4 \times 8$ sparse array $(77 \mathrm{GHz})$

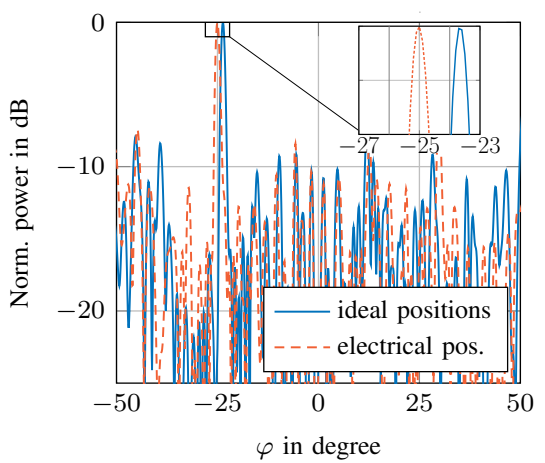

(c) $8 \times 8$ sparse array $(160 \mathrm{GHz})$

Fig. 7. DoA measurement results for the $4 \times 8$ ULA and the $4 \times 8$ sparse array at $77 \mathrm{GHz}$ and the $8 \times 8$ sparse array at $160 \mathrm{GHz}$ for a target at $\varphi=-25^{\circ}$. The results are determined both for ideal and electrical antenna positions derived from the proposed calibration method. Using electrical antenna positions there are lower deviations from the true DoA as can be seen in the enlarged subplot.

by the phase offset $\psi_{k}$. To process the calibrated electrical positions, the fractional values of $k^{\prime}$ have to be used. To enhance the DFT resolution resulting from the number of the spatial samples $N_{\mathrm{VX}}$, the indexes $n$ and the corresponding angles $\varphi_{n}$ can be calculated according to

$$
\begin{gathered}
n \in\left[\sin \left(\varphi_{\max }\right) \frac{-90^{\circ}}{\Delta \varphi}, \sin \left(\varphi_{\max }\right) \frac{90^{\circ}}{\Delta \varphi}\right] \cap \mathbb{Z}, \\
\text { and } \quad \varphi_{n}=\arcsin \left(n \frac{\Delta \varphi}{90^{\circ}}\right),
\end{gathered}
$$

with the desired angular resolution $\Delta \varphi$ and the angular field of view $\pm \sin \varphi_{\max } . N_{\mathrm{ZP}}$ in (8) is the overall number of Fourier samples according to the desired angular resolution.

\section{DoA Measurement Results}

The impact of an electrical antenna position shift is now evaluated for the ULA and both sparse arrays for a true DoA of $\varphi=-25^{\circ}$ in comparison. The DoA estimation is evaluated for both the ideal antenna element positions and the electrical element positions obtained from the calibration and using a DFT as shown in Section V. Comparing the ideal and electrical antenna positions, there is only a marginal difference between the true and the estimated DoA for the ULA, see Fig. 7(a). However, the sparse arrays are affected by the non-idealities and the coupling effects, see Fig. 7(b) and (c). The true DoA can only be estimated correctly, if the electrical antenna positions are used. For the $77 \mathrm{GHz}$ sparse array with PCB-antennas, the DoA estimation error is $0.25^{\circ}$. For the $160 \mathrm{GHz}$ sparse array the increased deviations in the electrical antenna positions lead to a significant DoA estimation error of $1.4^{\circ}$, if ideal antenna positions are assumed. Using the proposed calibration, the true DoA is found in all considered cases with an error of less than $0.1^{\circ}$.

\section{VII.CONCLUSION}

In this paper, the effect of non-idealities and coupling on the electrical antenna element positions is theoretically derived and proven by simulation and measurements using two MIMO radars. To determine the electrical antenna positions, a fast and practical calibration method is introduced. The method is robust to determine the true DoA and mitigates coupling and manufacturing tolerances, which are more relevant for a radar setup with increased frequencies and using integrated on-chip antennas on multiple chips as shown with an $8 \times 8$ array at $160 \mathrm{GHz}$.

\section{ACKNOWLEDGMENT}

This work was funded by the German Research Foundation with the project number 317632307.

\section{REFERENCES}

[1] J. Li and P. Stoica, "MIMO Radar with Colocated Antennas," IEEE Signal Processing Magazine, vol. 24, no. 5, pp. 106-114, Sep. 2007.

[2] I. Bekkerman and J. Tabrikian, "Target Detection and Localization Using MIMO Radars and Sonars," IEEE Transactions on Signal Processing, vol. 54 , no. 10 , pp. 3873-3883, Oct. 2006.

[3] C. Vasanelli et al., "Optimization of a MIMO Radar Antenna System for Automotive Applications," in 11th European Conference on Antennas and Propagation (EUCAP), Mar. 2017, pp. 1113-1117.

[4] M. Viberg and C. Engdahl, "Element Position Considerations for Robust Direction Finding Using Sparse Arrays," in Conference Record of the Thirty-Third Asilomar Conference on Signals, Systems, and Computers (Cat. No.CH37020), vol. 2, Oct. 1999, pp. 835-839.

[5] R. Feger et al., "Sparse Antenna Array Design and combined Range and Angle Estimation for FMCW Radar Sensors," in IEEE Radar Conference, May 2008, pp. 1-6.

[6] B. T. Arnold and M. A. Jensen, "The Effect of Antenna Mutual Coupling on MIMO Radar System Performance," IEEE Transactions on Antennas and Propagation, pp. 1-1, 2018.

[7] J.-G. Hong et al., "Compensation of Mutual Coupling in an Antenna Array for Direction of Arrival Estimation," in 15th International Conference on Advanced Communications Technology (ICACT), Jan. 2013, pp. 599-603.

[8] B. Friedlander and A. J. Weiss, "Direction Finding in the Presence of Mutual Coupling," IEEE Transactions on Antennas and Propagation, vol. 39, no. 3, pp. 273-284, Mar. 1991.

[9] B. Clerckx et al., "Impact of Antenna Coupling on $2 \times 2$ MIMO Communications," IEEE Transactions on Vehicular Technology, vol. 56, no. 3, pp. 1009-1018, May 2007. 